Tôhoku Math. Journ.

24 (1972). 167-190.

\title{
CORRESPONDENCE BETWEEN SUBGROUPS AND SUBALGEBRAS IN A CROSS PRODUCT VON NEUMANN ALGEBRA
}

\author{
Dedicated to Professor Gen-ichirô Sunouchi on his 60th birthday
}

\section{YoSHINORI HAGA AND ZIRô TAKEDA}

(Received Jan. 10, 1971, Revised Dec. 12, 1971)

Introduction. Observing the close analogy between theories of classical simple algebras and of continuous finite factors, M. Nakamura and Z. Takeda ([12], [13], [16] and [17]) established the Galois theory for finite factors. The main theorem of their theory is the following

THEOREM A. Let $\mathscr{A}$ be a continuous finite factor acting standardly on a separable Hilbert space $\mathscr{H}$, let $G$ be a countable group of outer automorphisms of $\mathscr{A}$ and let $\mathscr{B}$ be the subfactor of $\mathscr{A}$ consisting of all elements invariant under $G$. Suppose that the commutant $\mathscr{B}^{\prime}$ of $\mathscr{B}$ is finite. Then, the lattices of all subgroups of $G$ and of all intermediate subfactors between $\mathscr{A}$ and $\mathscr{B}$ are dually isomorphic under the Galois correspondence which carries a subgroup $F$ to an intermediate subfactor $\mathscr{C}$ invariant under $F$ in element-wise.

On the other hand, H. A. Dye introduced the notion of full group for a group of automorphisms of an abelian von Neumann algebra, and showed, among others, the next theorem, ([8; Proposition 6.1], also cf. [11; Theorem 3]).

Theorem B. Let $\mathscr{C l}$ be a von Neumann algebra with a faithfull normal trace $\tau$. Let $\mathscr{A}$ be a regular maximal abelian self-adjoint subalgebra of $\mathscr{C}$ having no minimal non-zero projections, and let $\mathscr{N}(\mathscr{A})$ be the collection of all unitary operators $U$ in $\mathscr{M}$ such that $U \mathscr{A} U^{*}=$ A. Denote by $G$ the group of all automorphisms $\phi_{U}(U \in \mathscr{N}(\mathscr{A}))$ of $\mathscr{A}$ where $\phi_{U}(A)=U A U^{*}$. Then there is a one-to-one correspondence between full subgroups $K$ of $G$ and intermediate von Neumann subalgebras $\mathscr{N}$ such that $\mathscr{A} \subset \mathscr{N} \subset \mathscr{M}$, obtained by associating with each full subgroup $K$ the intermediate subalgebra $\mathscr{R}\left[U \mid \phi_{U} \in K\right]$ and with each intermediate subalgebra $\mathscr{N}$ the subgroup $\left[\phi_{U} \mid U \in \mathscr{N}(\mathscr{A}) \cap \mathscr{N}\right]$.

In Theorem A, $\mathscr{A}$ indicates a factor, while in Theorem B it indicates an abelian self-adjoint algebra. These are the two extreme cases of von Neumann algebras. Nevertheless, making use of the notion of the cross 
product of von Neumann algebra, we may find that the Galois correspondence and the Dye correspondence are essentially of the same character. In this paper we investigate these correspondences for general finite von Neumann algebras $\mathscr{A}$ and groups of automorphisms of $\mathscr{A}$. For this purpose, Kallman's notion of generalized free action (Definition 1) of automorphisms of $\mathscr{A}$ is effective. It unifies the notion of outer automorphisms of factor $\mathscr{A}$ and that of free action of automorphisms of abelian $\mathscr{A}$. Besides, generalizing Dye's notion of absolute fixedness of automorphisms of abelian $\mathscr{A}$, we introduce the notion of locally inner automorphisms of general $\mathscr{A}$ (Definition 2), and by it we extend the notion of full group [G] determined by a group $G$ of automorphisms of $\mathscr{A}$ (Definition 3). Then the idea of H. Choda [3] makes it possible to characterize the elements of a full group $[G]$ as inner automorphisms of the cross product $G \otimes \mathscr{A}$ (Theorem 1). Using this characterization, we extend the Dye correspondence (Theorem 2) and as its dual relation, the Galois correspondence to general von Neumann algebras having finite commutants (Theorem 3). Recently M. Henle [9] has extended the theory of NakamuraTakeda to general von Neumann algebras. We shall consider his theory from our point of view in the last section.

Kindly enough, Prof. M. Nakamura, Prof. H. Choda and their colleagues have taken the pains of reading our manuscript carefully and giving us valuable comments. We wish here to express our thanks to them. Also we must acknowledge to Dr. M. Henle for the opportunity to see pre-publication copy of his paper, to which we are indebted deeply.

Notations. In the following, $\mathscr{A}$ is a von Neumann algebra acting on a separable Hilbert space, and $\mathscr{Z}$ is the center of $\mathscr{A}$. $\mathscr{A}_{p}\left(\right.$ resp. $\left.\mathscr{A}_{u}\right)$ is the set of all projections (resp. unitaries) of $\mathscr{A}$. The elements of $\mathscr{A}$ are denoted by capital letters, in particular, the indentity by $I$. For $A \in \mathscr{A}, C(A)$ denotes the central support of $A$ ([6; p. 7]). Automorphism means *-automorphism throughout this paper. $G$ is a group of automorphisms of $\mathscr{A}$ and the elements of $G$ are denoted by small letters, in particular, the unity by 1 . Automorphisms which are not necessarily in $G$ are denoted by Greek letters.

1. Free action and full group. In [15] J. von Neumann defined the notion of free action and applied it to the construction of certain factors. Given an abelian von Neumann algebra $\mathscr{A}$ and an automorphism $\alpha$ of $\mathscr{A}$, he defined $\alpha$ to be freely acting on $\mathscr{A}$ if, for every non-zero projection $P$ in $\mathscr{A}$, there exists a non-zero projection $Q$ in $\mathscr{A}$ satisfying $Q \leqq P$ and $\alpha(Q) Q=0$. Following H. A. Dye [7] a projection $P$ in $\mathscr{A}$ is abso- 
lutely fixed under $\alpha$ if $\alpha(Q)=Q$ for each $Q \leqq P$. Every automorphism $\alpha$ of $\mathscr{A}$ determines a maximal projection $F_{\alpha}$ absolutely fixed under it and $\alpha$ is freely acting on $\mathscr{A}$ if and only if $F_{\alpha}=0$. R. R. Kallman [10] extended this notion to general von Neumann algebras as follows.

Definition 1. Let $\mathscr{A}$ be a von Neumann algebra and $\alpha$ an automorphism of $\mathscr{A}$. $\alpha$ is said to be freely acting on $\mathscr{A}$ when

$$
A B=\alpha(B) A \quad \text { for all } B \in \mathscr{A}
$$

implies $A=0$.

When $\mathscr{A}$ is abelian, this definition agrees with the preceding one. When $\mathscr{A}$ is a factor, a freely acting automorphism is nothing but an outer automorphism. As Kallman noticed if $\alpha$ is freely acting on the center $\mathscr{Z}$, it is freely acting on $\mathscr{A}$ itself. Freely acting automorphisms and inner automorphisms are complementary types of automorphisms in the following sense.

Theorem (Kallman). Let $\mathscr{A}$ be a von Neumann algebra and $\alpha$ an automorphism of $\mathscr{A}$. Then $\mathscr{A}=\mathscr{A}_{1} \oplus \mathscr{A}_{2}, \alpha=\alpha_{1} \oplus \alpha_{2}, \alpha_{i}\left(A_{i}\right)=A_{i}$, $(i=1,2), \alpha_{1}$ is inner on $\mathscr{A}_{1}$ and $\alpha_{2}$ is freely acting on $\mathscr{A}_{2}$. This decomposition is unique.

All these facts are shown in [10]. A group $G$ of automorphisms of $\mathscr{A}$ is said to be freely acting if each $g \neq 1$ in $G$ is freely acting.

Next, we generalize the notion of absolute fixedness as follows.

Definition 2. Let $\alpha$ be an automorphism of $\mathscr{A}$ and $P$ be a central projection. Then $\alpha$ is locally inner on $P$ if $\alpha$ is inner on $\left.\mathscr{A}\right|_{P}$, that is if there exists a partial unitary $V_{\alpha, P}$ in $\mathscr{A}$ such that

and

$$
V_{\alpha, P} V_{\alpha, P}^{*}=V_{\alpha, P}^{*} V_{\alpha, P}=P
$$

$$
\alpha(P B)=V_{\alpha, P} B V_{\alpha, P}^{*} \quad \text { for all } B \in \mathscr{A} .
$$

Clearly when $\mathscr{A}$ is abelian, $\alpha$ is locally inner on $P$ if and only if $P$ is absolutely fixed under $\alpha$. Thus local innerness is a natural non-abelian extension of absolute fixedness.

Lemma 1. Let $\alpha$ be locally inner on $P$. Then

(i) $\alpha(P)=P$.

(ii) if $\beta$ is locally inner on $Q \in \mathscr{Z}_{p}, \alpha \beta$ is locally inner on $P Q$.

(iii) $\alpha^{-1}$ is locally inner on $P$.

(iv) $\alpha$ is locally inner on $Q \in \mathscr{Z}_{p}$ such that $Q \leqq P$.

(v) if $\alpha$ is locally inner on another $Q \in \mathscr{Z}_{p}, \alpha$ is locally inner on 
$P \vee Q$. This fact holds for a join of infinitely many central projections.

Proof. We prove only (v). $P \vee Q=P+(Q-P Q)$ and $\alpha$ is locally inner on $Q-P Q$ by (iv). Hence we may assume $P Q=0$. Then we may take $V_{\alpha, P+Q}=V_{\alpha, P}+V_{\alpha, Q}$. For infinitely many central projections it is sufficient to consider the limit of finite sums in the strong operator topology (cf. [10; Lemma 1.9]).

Lemma 2. If an automorphism $\alpha$ of $\mathscr{A}$ satisfies (1) for a given $A \in \mathscr{A}$, then $\alpha$ is locally inner on $C(A)$.

Proof. Let $A=W|A|$ be the polar decomposition of $A$, and put $P=C(A)$. Following the proof of [10; Theorem 1.1], we see that $W W^{*}=$ $W^{*} W=P$ and, for all $B \in \mathscr{A}, W B=\alpha(B) W$ or $W B=W P B=\alpha(P B) W$. That is, $W B W^{*}=\alpha(P B)$. Therefore $\alpha$ is locally inner on $P$.

By Lemma $1(\mathrm{v})$, there exists the unique maximal central projection $F_{\alpha}$ on which $\alpha$ is locally inner. We simply write $V_{\alpha}$ instead of $V_{\alpha, F}$. Then, in parallel with Dye's definition of the free action, we get the following corollary which is a different version of Kallman's theorem quoted above.

CoRollary. $\alpha$ is freely acting on $A$ if and only if $F_{\alpha}=0$.

Now, put $F(\alpha, \beta)=F_{\alpha^{-1 \beta}}$. Then,

$$
F(\alpha, \beta)=F(\beta, \alpha) .
$$

By (2)

$$
\beta(A)=\alpha\left(V_{\alpha^{-1 \beta}} A V_{\alpha^{-1 \beta}}^{*}\right) \quad \text { if } \quad C(A) \leqq F(\alpha, \beta) .
$$

In particular,

$$
\alpha(F(\alpha, \beta))=\beta(F(\alpha, \beta)) .
$$

Definition 3. Let $G$ be a group of automorphisms of $\mathscr{A}$ and denote by $[G]$ the collection of all automorphisms $\alpha$ of $\mathscr{A}$ such that

$$
\sup _{g \in G} F(\alpha, g)=I \text {. }
$$

$[G]$ is called the full group determined by $G$. A group $G$ is called full if $G=[G]$ (cf. [7]).

Lemma 3. Let $G$ be a group of automorphisms of $\mathscr{A}$. Then,

(i) $[G]$ is again a group of automorphisms of $\mathscr{A}$.

(ii) $[[G]]=[G]$. 
(iii) Elements $\alpha$ of $[G]$ are precisely those endomorphisms of $\mathscr{A}$ having a representation

$$
\alpha(A)=\sum_{n} P_{n} g_{n}\left(V A V^{*}\right) \quad \text { for all } A \in \mathscr{A},
$$

where $g_{n} \in G, V \in \mathscr{A}_{u}$, and $\left\{P_{n}\right\}$ (resp. $\left.\left\{g_{n}^{-1}\left(P_{n}\right)\right\}\right)$ is a family of mutually orthogonal central projections having sum $I$.

(iv) If $\mathscr{A s}$ finite with a faithful normal trace $\tau$ and every element of $G$ preserves $\tau$, then each element $\alpha$ of $[G]$ preserves $\tau$ also.

Proof. (i), (ii) and (iii) are proved by easy modifications of [7; Lemma 3.1] so we omit the proofs. (iv). For $\alpha \in[G]$ and non-negative $A \in \mathscr{A}$,

$$
\begin{aligned}
\tau(\alpha(A)) & =\tau\left(\alpha\left[A \sup _{g \in G} F(\alpha, g)\right]\right)=\sup _{g} \tau(\alpha[A F(\alpha, g)]) \\
& =\sup _{g} \tau(g[A F(\alpha, g)])=\sup _{g} \tau(A F(\alpha, g)) \\
& =\tau\left(A \sup _{g} F(\alpha, g)\right)=\tau(A)
\end{aligned}
$$

In a representation such as (3) of an automorphism $\alpha$ of [G], we omit henceforth to write the supplementary conditions on $V$ and $\left\{P_{g}\right\}$ to avoid the trouble of the statement.

We notice here that if $G$ is freely acting on $\mathscr{A}$ and $\alpha \in[G]$, then

$$
F(\alpha, g) F(\alpha, h) \leqq F(g, h)=0 \quad \text { for } g \neq h,
$$

and so

$$
\sum_{g} F(\alpha, g)=\sup _{g} F(\alpha, g)=I \text {. }
$$

Each $P_{n}$ in (3) coincides with $\alpha\left(F\left(\alpha, g_{n}\right)\right)$.

2. Cross product. Let $\mathscr{A}$ be a von Neumann algebra acting on a separable Hilbert space $\mathscr{H}$ and $G$ a countable group of automorphisms of $\mathscr{A}$. Let $G \otimes \mathscr{H}$ be the set of all formal sums $\sum_{a \in G} a \otimes \xi_{a}\left(\xi_{a} \in \mathscr{H}\right)$ for which $\sum_{a}\left\|\xi_{a}\right\|^{2}<\infty$. Define addition and scalar multiplication on $G \otimes \mathscr{H}$ by

$$
\left(\sum a \otimes \xi_{a}\right)+\left(\sum a \otimes \eta_{a}\right)=\sum a \otimes\left(\xi_{a}+\eta_{a}\right)
$$

and

$$
\lambda\left(\sum a \otimes \xi_{a}\right)=\sum a \otimes \lambda \xi_{a}
$$

where $\lambda$ is a complex number (cf. [20]). Then $G \otimes \mathscr{H}$ becomes a separable Hilbert space with an inner product

$$
\left\langle\sum a \otimes \xi_{a} \mid \sum a \otimes \eta_{a}\right\rangle=\Sigma\left\langle\xi_{a} \mid \eta_{a}\right\rangle .
$$


Define an operator $g \otimes A(g \in G, A \in \mathscr{A})$ on $G \otimes \mathscr{H}$ by

$$
(g \otimes A)\left(\sum a \otimes \xi_{a}\right)=\sum_{a \in G} a g^{-1} \otimes a(A) \xi_{a} .
$$

Then, direct computations show that

$$
(g \otimes A)(h \otimes B)=g h \otimes h^{-1}(A) B
$$

and

$$
(g \otimes A)^{*}=g^{-1} \otimes g\left(A^{*}\right) .
$$

The cross product $G \otimes \mathscr{A}$ is defined as the von Neumann algebra on $G \otimes \mathscr{H}$ generated by $\{g \otimes A \mid g \in G, A \in \mathscr{A}\}$, where

$$
1 \otimes \mathscr{A}=\{1 \otimes A \mid A \in \mathscr{A}\}
$$

is a von Neumann subalgebra of $G \otimes \mathscr{A}$ and it is algebraically isomorphic to $\mathscr{A}$. Hereafter we shall identify $A \in \mathscr{A}$ with $1 \otimes A \in 1 \otimes \mathscr{A}$ when we have no fear to lead to confusion. $g \otimes I(g \in G)$ is a unitary operator in $G \otimes \mathscr{A}$, and it induces the automorphism $g$ on $\mathscr{A}$ :

$$
(g \otimes I)(1 \otimes A)(g \otimes I)^{*}=(g \otimes A)\left(g^{-1} \otimes I\right)=1 \otimes g(A) .
$$

It is known that the cross product $G \otimes \mathscr{A}$ is independent of the particular representation of $\mathscr{A}$ used to construct it $([21 ; 8.6])$.

Next we review the concept of expectation briefly which we shall use heavily in the next section (see [1] and [19]).

Let $\mathscr{B}$ be a von Neumann subalgebra of $\mathscr{A}$. An expectation from $\mathscr{A}$ on $\mathscr{B}$ is a positive $*$-linear mapping $\Phi_{\mathscr{A}}$ of $\mathscr{A}$ into $\mathscr{B}$ such that $\Phi_{\mathscr{B}}(I)=I$ and

$$
\Phi_{\mathscr{B}}(B A)=B \Phi_{\mathscr{B}}(A) \quad \text { for } A \in \mathscr{A}, B \in \mathscr{B} .
$$

By taking adjoints, it follows immediately that

$$
\Phi_{\mathscr{O}}(A B)=\Phi_{\mathscr{F}}(A) B \text {. }
$$

It satisfies

$$
\Phi_{\mathscr{O}}\left(A^{*} A\right) \geqq \Phi_{\mathscr{\mathscr { A }}}(A)^{*} \Phi_{\mathscr{O}}(A) .
$$

$\mathscr{B}$ is precisely the set of fixed points of $\Phi_{\mathscr{B}}$.

In particular, when $\mathscr{A}$ is a finite algebra with a faithful normal trace $\tau$ and $\mathscr{B}$ is an arbitrary von Neumann subalgebra of $\mathscr{A}$, it is well known that there exists a uniquely determined faithful normal expectation from $\mathscr{A}$ on $\mathscr{B}$ such that

$$
\tau\left(\Phi_{\mathscr{O}}(A) B\right)=\tau(A B)
$$
for $B \in \mathscr{B}$.

We call this the natural expectation from $\mathscr{A}$ on $\mathscr{B}$. The trace norm 
of $A \in \mathscr{A}$ is defined by $\|A\|_{2}=\left(\tau\left(A^{*} A\right)\right)^{1 / 2}$, then by (5) this norm satisfies $\left\|\Phi_{\mathscr{S}_{B}}(A)\right\|_{2} \leqq\|A\|_{2}$.

In the case of cross product $G \otimes \mathscr{A}$, for $T \in G \otimes \mathscr{A}$, we may put

$$
\Phi(T)=\sum_{g \in G} P_{g} T P_{g}
$$

where $P_{g}$ are the projections of $G \otimes \mathscr{C}$ onto $g \otimes \mathscr{H}$, and the sum is taken in the strong topology. Then, $\Phi$ is a faithful normal expectation of $G \otimes \mathscr{A}$ onto $\mathscr{A}$ (see [1; Appendix]). Notice that this expectation does not presuppose the finiteness of $\mathscr{A}$. A normal state $p$ of $\mathscr{A}$ is extended to $G \otimes \mathscr{A}$ by $p \circ \Phi$. In particular, when $\mathscr{A}$ is finite and there is a faithful normal $G$-invariant trace $\tau, \tau$ is extended to the faithful normal trace on $G \otimes \mathscr{A}$. Hence, in this case, $G \otimes \mathscr{A}$ is also finite. Using the state $p$ on $\mathscr{A}$ and the extended state $p$ on $G \otimes \mathscr{A}$, we may define Hilbert norms on $\mathscr{A}$ and $G \otimes \mathscr{A}$ by $\|T\|_{p}=\left(p\left(T^{*} T\right)\right)^{1 / 2}$ for $T \in \mathscr{A}$ and $T \in G \otimes \mathscr{A}$ respectively. Let $\mathscr{K}$ be the completion of pre-Hilbert space $\mathscr{A}$. $\mathscr{K}$ is the Gelfand-Segal representation space of $\mathscr{A}$ by the state $p$, and the completion of the pre-Hilbert space $G \otimes \mathscr{A}$ is that of $G \otimes \mathscr{A}$ by the extended $p$, which may be identified with $G \otimes \mathscr{K}$, [18]. Since every element of $G \otimes \mathscr{A}$ can be considered as an element of $G \otimes \mathscr{K}$, every $T \in G \otimes \mathscr{A}$ has the Fourier expansion

$$
T=\sum_{g \in G} g \otimes T_{g} \quad\left(T_{g} \in K\right)
$$

converging in $p$-norm, and a direct computation shows that

$$
T_{g}=\Phi\left(\left(g^{-1} \otimes I\right)\left(\sum_{h} h \otimes T_{h}\right)\right) \in \mathscr{A} \quad \text { for all } g \in G \text {. }
$$

In [3] H. Choda characterized the elements of full group [G] as the inner automorphisms of $G \otimes \mathscr{A}$, when $\mathscr{A}$ is an abelian von Neumann algebra. We generalize this result to non-abelian von Neumann algebras. Put

$$
\mathscr{U}(G \otimes \mathscr{A}, \mathscr{A})=\left\{U \in(G \otimes \mathscr{A})_{u} \mid U \mathscr{A} U^{*}=\mathscr{A}\right\}
$$

Then, every element of $\mathscr{U}(G \otimes \mathscr{A}, \mathscr{A})$ induces an automorphism of $\mathscr{A}$.

THeOREM 1. Let $\mathscr{A}$ be a von Neumann algebra, $G$ a countable discrete group of automorphism acting freely on $\mathscr{A}$, and $\alpha$ an automorphism in $[G]$. Then $\alpha$ can be extended to an inner automorphism of $G \otimes \mathscr{A}$. Conversely, an automorphism of $\mathscr{A}$ induced by $U \in \mathscr{Q}_{2}^{\prime}(G \otimes \mathscr{A}, \mathscr{A})$ belongs to $[G]$.

Proof. Let $P_{g}=F(\alpha, g) \in \mathscr{\varkappa}_{p}$ for $g \in G$. Then, if $g \neq h$,

$$
P_{g} P_{h}=F(\alpha, g) F(\alpha, h) \leqq F(g, h)=0
$$


by the free action of $G$. That is, $\left\{P_{g}\right\}$ is a family of mutually orthogonal central projections. Since $\alpha \in[G]$,

$$
\sum_{g} P_{g}=\sum_{g} F(\alpha, g)=I \text {. }
$$

Put

$$
U=\sum_{g} g \otimes V_{\alpha^{-1} g}^{*}
$$

Then the following direct computations show that $U$ is a unitary operator in $G \otimes \mathscr{A}$ and that $U$ induces an inner automorphism of $G \otimes \mathscr{A}$ which is an extension of $\alpha$.

$$
\begin{aligned}
U U^{*} & =\left(\sum_{g} g \otimes V_{\alpha^{-1} g}^{*}\right)\left(\sum_{h} h^{-1} \otimes h\left(V_{\alpha^{-1} h}\right)\right) \\
& =\sum_{g, h} g h^{-1} \otimes h\left(V_{\alpha^{-1} g}^{*} V_{\alpha^{-1}}\right)=1 \otimes \sum_{g} g\left(V_{\alpha^{-1} g}^{*} V_{\alpha^{-1} g}\right) \\
& =1 \otimes \sum_{g} g(F(\alpha, g))=1 \otimes \sum_{g} \alpha(F(\alpha, g))=I, \\
U^{*} U & =\left(\sum_{g} g^{-1} \otimes g\left(V_{\alpha^{-1} g}\right)\right)\left(\sum_{g} h \otimes V_{\alpha^{-1} h}^{*}\right) \\
& =\sum_{g, h} g^{-1} h \otimes h^{-1} g\left(V_{\alpha^{-1} g}\right) V_{\alpha^{-1} h}^{*} \\
& =\sum_{g, h} g^{-1} h \otimes h^{-1} \alpha\left(V_{\alpha^{-1} g} V_{\alpha^{-1} g} V_{\alpha^{-1} g}^{*}\right) h^{-1} \alpha\left(V_{\alpha^{-1} h} V_{\alpha^{-1} h}^{*} V_{\alpha^{-1} h}^{*}\right) \\
& =\sum_{g, h} g^{-1} h \otimes h^{-1} \alpha\left(V_{\alpha^{-1} g} F(\alpha, g) F(\alpha, h) V_{\alpha^{-1} h}^{*}\right) \\
& =1 \otimes \sum_{g} g^{-1} \alpha(F(\alpha, g))=1 \otimes \sum_{g} F(\alpha, g)=I
\end{aligned}
$$

and

$$
\begin{aligned}
U(1 \otimes A) U^{*} & =\left(\sum_{g} g \otimes V_{\alpha^{-1} g}^{*} A\right)\left(\sum_{h} h^{-1} \otimes h\left(V_{\alpha^{-1} h}\right)\right) \\
& =\sum_{g, h} g h^{-1} \otimes h\left(V_{\alpha^{-1} g}^{*} A V_{\alpha^{-1} h}\right) \\
& =\sum_{g, h} g h^{-1} \otimes \alpha\left(V_{\alpha^{-1} h} V_{\alpha^{-1} g}^{*} A V_{\alpha^{-1} h} V_{\alpha^{-1} h}^{*}\right) \\
& =1 \otimes \sum_{g} \alpha(F(\alpha, g) A)=1 \otimes \alpha(A) .
\end{aligned}
$$

Conversely, let $\alpha$ be the automorphism of $\mathscr{A}$ induced by

$$
U=\sum_{g} g \otimes A_{g} \in \mathscr{U}(G \otimes \mathscr{A}, \mathscr{A}) \text {. }
$$

Since $U(1 \otimes B)=(1 \otimes \alpha(B)) U$ for all $B \in \mathscr{A}$,

$$
\sum_{g} g \otimes A_{g} B=\sum_{g} g \otimes g^{-1} \alpha(B) A_{g},
$$

hence, $A_{g} B=g^{-1} \alpha(B) A_{g}$ for all $B \in \mathscr{A}$ and $g \in G$. Then by Lemma 2, $g^{-1} \alpha$ is locally inner on the central support $P_{g}$ of $A_{g}$. Hence

$$
P_{g} \leqq F(\alpha, g) \text {. }
$$

If $g \neq h$, then $P_{g} P_{h} \leqq F(\alpha, g) F(\alpha, h) \leqq F(g, h)=0$. Therefore $\left\{P_{g}\right\}$ is a 
family of mutually orthogonal central projections. As $U$ is unitary,

$$
1 \otimes I=U^{*} U=\sum_{g, h} g^{-1} h \otimes h^{-1} g\left(A_{g}^{*}\right) A_{h} \text {. }
$$

Hence

$$
\sum_{g} A_{g}^{*} A_{g}=I
$$

Since $P_{g}$ coincides with the central support of $A_{g}^{*} A_{g}$, we have $\sum_{g} P_{g}=I$. Therefore

$$
\sup _{g \in G} F(\alpha, g) \geqq \sum_{g} P_{g}=I
$$

hence, $\alpha \in[G]$.

By Lemma 3, the automorphism $\alpha \in[G]$ induced by $U \in \mathscr{U}(G \otimes \mathscr{A}, \mathscr{A})$ has a representation

$$
\alpha(A)=\sum_{g} P_{g} g\left(V A V^{*}\right)
$$

From this and $U(1 \otimes A) U^{*}=1 \otimes \alpha(A)$ we get the following corollary, where $Q_{g}=g^{-1}\left(P_{g}\right)$.

CoRollary 1. Let $\mathscr{A}$ and $G$ be as in Theorem 1. Then any $U \in$ $\mathscr{U}(G \otimes \mathscr{A}, \mathscr{A})$ has a unique decomposition

$$
U=\left(\sum_{g} g \otimes Q_{g}\right)(1 \otimes V)
$$

where $V \in \mathscr{A}_{u},\left\{Q_{g}\right\}$ (resp. $\left.\left\{g\left(Q_{g}\right)\right\}\right)$ is a family of mutually orthogonal central projections having sum $I$. Conversely, any such $U \in G \otimes \mathscr{A}$ is in $\mathscr{C}(G \otimes \mathscr{A}, \mathscr{A})$.

This corollary has been proved by $H$. Behncke nuder the more restricted assumption that $G$ acts freely on the center $\mathscr{z}$, ([2; Lemma 2.1]).

CoRollary 2. If $\mathscr{A}$ is a factor, then every element of [G] coincides with an element of $G$ up to an inner automorphism.

This is a generalization of [14, Corollary].

The following lemma will be used sometimes later.

LEMma 4. If $G$ is freely acting on $\mathscr{A}$, we have

$$
(G \otimes \mathscr{A}) \cap(1 \otimes \mathscr{A})^{\prime}=1 \otimes \mathscr{z} .
$$

Proof. If $\sum_{g} g \otimes A_{g} \in G \otimes \mathscr{A}$ commutes with all $1 \otimes B$ in $1 \otimes \mathscr{A}$, then by

$$
\left(\sum_{g} g \otimes A_{g}\right)(1 \otimes B)=\sum_{g} g \otimes A_{g} B
$$

and 


$$
(1 \otimes B)\left(\sum_{g} g \otimes A_{g}\right)=\sum_{g} g \otimes g^{-1}(B) A_{g},
$$

we have

$$
A_{g} B=g^{-1}(B) A_{g} \quad \text { for all } B \in \mathscr{A} \text {. }
$$

Since $G$ is freely acting, we have

$$
A_{g}=0 \text { for } g \neq 1 \text { and } A_{1} \in \mathscr{Z} \text {. }
$$

Hence

$$
\sum_{g} g \otimes A_{g}=1 \otimes A_{1} \in 1 \otimes \mathscr{Z} .
$$

On the other hand, $1 \otimes \mathscr{Z} \subset(G \otimes \mathscr{A}) \cap(1 \otimes \mathscr{A})^{\prime}$ is obvious.

Corollary. If $G$ is freely acting on $\mathscr{A}$, then the center of $G \otimes \mathscr{A}$ is the subalgebra $\mathscr{Z}^{G}$ of fixed elements under $G$ in $\mathscr{Z}$.

In fact, $Z \in \mathscr{Z}$ commutes with every $g \otimes I$ if and only if $Z \in \mathscr{Z}^{a}$.

3. Full subgroups and subalgebras. In terms of the cross product, Theorem B in the introduction states that, for the cross product $G \otimes \mathscr{A}$ of an abelian von Neumann algebra $\mathscr{A}$, there exists a one-to-one correspondence between full subgroups of the full groups $[G]$ and intermediate subalgebras of $G \otimes \mathscr{A}$, that is, von Neumann subalgebras which contain $\mathscr{A}$. In this section, we generalize this result for finite von Neumann algebras. The way of the proof is quite similar to that of Dye [8].

Throughout this section, $\mathscr{A}$ is a finite von Neumann algebra with a faithful normal trace $\tau$, and $G$ is a countable discrete group of automorphisms of $\mathscr{A}$ acting freely and preserving $\tau$ invariant. Therefore $G \otimes \mathscr{A}$ is finite too, and the trace $\tau$ can be extended to $G \otimes \mathscr{A}$. Hence there exists a unique natural expectation of $G \otimes \mathscr{A}$ on every von Neumann subalgebra $\mathscr{B}$ of $G \otimes \mathscr{A}$ (see $\S 2$ ).

Let $\mathscr{U}(G \otimes \mathscr{A}, \mathscr{A})=\left\{U \in(G \otimes \mathscr{A})_{u} \mid U \mathscr{A} U^{*}=\mathscr{A}\right\}$ and denote by $\phi_{U}$ the automorphism of $\mathscr{A}$ induced by $U \in \mathscr{U}(G \otimes \mathscr{A}, \mathscr{A})$. Because $\mathscr{U}(G \otimes \mathscr{A}, \mathscr{A})$ includes $G \otimes I$ and $1 \otimes \mathscr{A}$, the von Neumann algebra $\mathscr{R}[\mathscr{U}(G \otimes \mathscr{A}, \mathscr{A})]$ generated by $\mathscr{U}(G \otimes \mathscr{A}, \mathscr{X})$ coincides with $G \otimes \mathscr{A}$. This corresponds to the fact that $\mathscr{A}$ is a regular maximal abelian self-adjoint subalgebra of $G \otimes \mathscr{A}$, when $\mathscr{A}$ is abelian (cf. [5]).

The mapping $U \rightarrow \phi_{U}$ is a homomorphism from $\mathscr{L}(G \otimes \mathscr{A}, \mathscr{A})$ into the group of all trace-preserving automorphisms of $\mathscr{A}$. Its kernel is the group $\mathscr{Z}_{u}$ of all unitaries in $\mathscr{Z}$ by Lemma 4 . The group

$$
\left[\phi_{U} \mid U \in \mathscr{L}(G \otimes \mathscr{A}, \mathscr{A})\right]
$$

coincides with the full group $[G]$ by Theorem 1, hence this group is full. 
Moreover, let $\mathscr{B}$ be an intermediate subalgebra of $G \otimes \mathscr{A}$, then

$$
K(\mathscr{B})=\left[\phi_{U} \mid U \in \mathscr{U}(G \otimes \mathscr{A}, \mathscr{A}) \cap \mathscr{B}\right]
$$

is a full subgroup of [G]. In fact, every element $\alpha$ of the full group determined by $K(\mathscr{B})$ has a form

$$
\alpha(A)=\sum_{n} P_{n} \gamma_{n}\left(V A V^{*}\right) \quad\left(\gamma_{n} \in K(\mathscr{B})\right) \cdot
$$

Then, if $\gamma_{n}$ is induced by $U_{n} \in \mathscr{U}(G \otimes \mathscr{A}, \mathscr{A}) \cap \mathscr{B}, \alpha$ is induced by the unitary $U=\sum_{n} U_{n}\left(1 \otimes \gamma_{n}^{-1}\left(P_{n}\right) V\right) \in \mathscr{U}(G \otimes \mathscr{A}, \mathscr{A}) \cap \mathscr{B}$.

LEMMA 5. Let $\mathscr{B}$ be an intermediate subalgebra of $G \otimes \mathscr{A}$, then the expectation $\Phi_{\mathscr{B}}(U)$ of $U \in \mathscr{C}(G \otimes \mathscr{A}, \mathscr{A})$ onto $\mathscr{B}$ is a partial isometry and its initial and final projections are both in $\mathscr{\varkappa}$.

Proof. (Cf. [8; Lemma 6.1] and [4]). Let $\Phi_{B}(U)=V S$ be the polar decomposition of $\Phi_{\mathscr{B}}(U)$. In view of Lemma 4 , if we follow the proof of [8; Lemma 6.1], we get $\phi_{U}(A) V S=V S A$. Hence

$$
A U^{*} V S=U^{*} \phi_{U}(A) V S=U^{*} V S A \quad \text { for all } A \in \mathscr{A} \text {. }
$$

Then, by Lemma 4 , we have $U^{*} V S \in \mathscr{Z} \subset \mathscr{B}$ and hence,

$$
U^{*} V S=\Phi_{\mathscr{B}}\left(U^{*} V S\right)=\Phi_{\mathscr{B}}\left(\left(U^{*} \Phi_{\mathscr{B}}(U)\right)=\Phi_{\mathscr{B}}\left(U^{*}\right) \phi_{\mathscr{B}}(U)=S^{2}\right. \text {. }
$$

Hence,

$$
S^{4}=S V^{*} U U^{*} V S=S V^{*} V S=\Phi_{\infty}(U)^{*} \Phi_{s}(U)=S^{2} .
$$

Therefore $S^{2}$ is a projection, and becauce $S$ is non-negative, $S$ is a projection also. Moreover, as $V^{*} V$ is the support of $S$, we have $S=V^{*} V$. Thus $\Phi_{\mathscr{B}}(U)=V S=V V^{*} V=V$ proving $\Phi_{\mathscr{B}}(U)$ is a partial isometry. The remained part is easily seen.

LEMмa 6. Let $\mathscr{B}$ be an intermediate subalgebra of $G \otimes \mathscr{A}$. Let $V \in \mathscr{B}$ be a partial isometry such that $V V^{*}, V^{*} V \in \mathscr{Z}, V \mathscr{A} V^{*} \subset \mathscr{A}$ and $V^{*} \mathscr{A} V \subset \mathscr{A}$. Then $V$ can be extended to a unitary operator $W$ in $\mathscr{U}(G \otimes \mathscr{A}, \mathscr{A}) \cap \mathscr{B}$.

Proof. The proof of this lemma is contained in the proof of [8; Lemma 6.1].

LEMMA 7. (Cf. [7; Lemma 3.4]). Let $\alpha$ be an arbitrary automorphism of $\mathscr{A}$, and $G$ a given group of automorphisms of $\mathscr{A}$. Then there exists a unique maximal central projection $E([G], \alpha)$ and $\beta$ in $[G]$ with the property that, for all $A$ in $\mathscr{A}$,

It holds

$$
E([G], \alpha) \alpha(A)=E([G], \alpha) \beta(A) .
$$




$$
E([G], \alpha)=\sup _{\gamma \in[G]} \alpha(F(\alpha, \gamma))=\alpha(F(\alpha, \beta)) .
$$

Proof. Following the proof of [7; Lemma 3.4], we get a maximal family $\left(P_{n}, \beta_{n}\right)$ such that $P_{n}$ are mutually orthogonal central projections, $\beta_{n} \in[G]$ and $\alpha^{-1}\left(P_{n}\right)=\beta_{n}^{-1}\left(P_{n}\right)$. Therefore the projections $\beta_{n}^{-1}\left(P_{n}\right)$ are mutually orthogonal. By Theorem 1 , each $\beta_{n} \in[G]$ can be extended to an inner automorphism of $G \otimes \mathscr{A}$ induced by some unitary $U_{n}$ in $\mathscr{C}(G \otimes \mathscr{A}, \mathscr{A})$. Set $V_{n}=U_{n} \beta_{n}^{-1}\left(P_{n}\right)$, then $V_{n}$ is a partial isometry such that $V_{n} V_{n}^{*}=P_{n}$ and $V_{n}^{*} V_{n}=\beta_{n}^{-1}\left(P_{n}\right)$. Therefore the operator $V=\sum_{n=1}^{\infty} V_{n}$ is a partial isometry such that $V^{*} V, V V^{*} \in \mathscr{\varkappa}$ and evidently $V \mathscr{A} V^{*} \subset \mathscr{A}$ and $V^{*} \mathscr{A} V \subset \mathscr{A}$. Then, by Lemma $6, V$ can be extended to a unitary operator $W$ in $\mathscr{U}(G \otimes \mathscr{A}, \mathscr{A})$. The automorphism $\beta$ of $\mathscr{A}$ induced by $W$ is in [G] by Theorem 1. Put $P=\sum_{n} P_{n}$, then we have

$$
\begin{aligned}
P \beta(A) & =\sum_{n} P_{n} W A W^{*}=\sum_{n} P_{n}\left(\sum_{m} V_{m}\right) A\left(\sum_{m} V_{m}\right)^{*} \\
& =\sum_{n} P_{n} V_{n} A V_{n}^{*}=\sum_{n} U_{n} \beta_{n}^{-1}\left(P_{n}\right) A \beta_{n}^{-1}\left(P_{n}\right) U_{n}^{*} \\
& =\sum_{n} P_{n} \beta_{n}(A)=\sum_{n} P_{n} \alpha(A)=P \alpha(A) .
\end{aligned}
$$

This projection $P=E([G], \alpha)$ has the required properties. It is shown again by analogous discussions to [7; Lemma 3.4].

LEMMA 8. (Cf. [8; Lemma 6.1]. Let $\mathscr{B}$ be an intermediate subalgebra of $G \otimes \mathscr{A}$, and let $K(\mathscr{B})$ be the full group of automorphisms of $\mathscr{A}$ induced by $\mathscr{U}(G \otimes \mathscr{A}, \mathscr{A}) \cap \mathscr{B}$. Then for each $U$ in $\mathscr{U}(G \otimes \mathscr{A}, \mathscr{A})$, there exists a $W$ in $\mathscr{U}(G \otimes \mathscr{A}, \mathscr{L}) \cap \mathscr{B}$ such that

$$
\Phi_{\mathscr{B}}(U)=E\left(K(\mathscr{B}), \phi_{U}\right) W .
$$

Proof. We have already remarked that the subgroup $K=K(\mathscr{B})$ is is full. Applying Lemma 5, it follows that the operator $V=\Phi_{\mathscr{B}}(U)$ is a partial isometry such that $E=V^{*} V$ and $F=V V^{*}$ are both in $\mathscr{z}$. $V A V^{*}=\Phi_{\mathscr{B}}(U) A V^{*}=\Phi_{\mathscr{B}}\left(\phi_{U}(A) U\right) V^{*}=\phi_{U}(A) V V^{*}=\phi_{U}(A) F \in \mathscr{A}$ for all $A \in \mathscr{A}$. Hence $V \mathscr{A} V^{*} \subset \mathscr{A}$. Similarly $V^{*} \mathscr{A} V \subset \mathscr{A}$. Therefore, by Lemma $6, V$ can be extended to a unitary $W$ in $\mathscr{U}(G \otimes \mathscr{A}, \mathscr{A}) \cap \mathscr{B}$. It follows from $V=\Phi_{\mathscr{B}}(U)=F W$ that

$$
F \phi_{U}(A)=V A V^{*}=F W A W^{*} F=F \phi_{W}(A)
$$

for all $A \in \mathscr{A}$, so that $F \leqq E\left(K, \phi_{U}\right)$.

On the other hand, writing $E_{1}=E\left(K, \phi_{U}\right)$, we have by Lemma 7 that there exists a $\phi_{W}$ in $K$ such that $E_{1} \phi_{W}(A)=E_{1} \phi_{U}(A)$, for all $A \in \mathscr{A}$. Substituting $W^{*} A W \in \mathscr{A}$ for $A$, we have $E_{1} A U W^{*}=E_{1} U W^{*} A$. Since $E_{1}$ is in $\mathscr{Z}$, it follows that $E_{1} U W^{*}$ commutes with any $A \in \mathscr{A}$ and we deduce $E_{1} U W^{*} \in \mathscr{Z}$ by Lemma 4. Evidently, $E_{1} U W^{*}$ is a partial isometry 
and satisfies all the properties of Lemma 6 , so it can be extended to a unitary $W^{\prime}$ in $\mathscr{C}(G \otimes \mathscr{A}, \mathscr{A}) \cap \mathscr{B}$. Then $E_{1} U W^{*}=E_{1} W^{\prime}$ and $W^{\prime} W \in$ $\mathscr{U}(G \in \mathscr{A}, \mathscr{A}) \cap \mathscr{B}$. Hence $E_{1} U=E_{1} W^{\prime} W \in \mathscr{B}$. Therefore

$$
\begin{aligned}
(I-F) E_{1} U & =(I-F) \Phi_{\mathscr{B}}\left(E_{1} U\right) \\
& =(I-F) E_{1} \Phi_{\mathscr{B}}(U)=(I-F) E_{1} F W=0 .
\end{aligned}
$$

As $U$ is unitary, this shows that $(I-F) E_{1}=0$, or $E_{1} \leqq F$. Accordingly, $F=E\left(K, \phi_{U}\right)$.

CoRollary 1. Every intermediate subalgebra $\mathscr{B}$ of $G \otimes \mathscr{A}$ is generated by $\mathscr{U}(G \otimes \mathscr{A}, \mathscr{A}) \cap \mathscr{B}$.

Proof. Let $\mathscr{B}_{1}$ denote the von Neumann subalgebra of $\mathscr{B}$ generated by $\mathscr{U}(G \otimes \mathscr{A}, \mathscr{A}) \cap \mathscr{B}$. Then the above lemma shows that, for each $U$ in $\mathscr{U}(G \otimes \mathscr{A}, \mathscr{A}), \Phi_{\mathscr{B}}(U)=F W \in \mathscr{B}_{1}$. As $G \otimes \mathscr{A}$ is generated by $\mathscr{U}(G \otimes \mathscr{A}, \mathscr{A})$, it follows that $\Phi_{\mathscr{B}}(A) \in \mathscr{B}_{1}$ for all $A \in G \otimes \mathscr{A}$. Hence $\mathscr{B}=\mathscr{B}_{1}$.

COROLlaRY 2. $\phi_{U}$ is freely acting on $\mathscr{A}$, if and only if $\Phi_{\mathscr{N}}(U)=0$.

Proof. Apply the lemma to the case $\mathscr{B}=\mathscr{A}$ and note that $K(\mathscr{A})$ is the set of all inner automorphisms.

Theorem 2. (Cf. [8; Proposition 6.1]). Let $\mathscr{A}$ be a finite von Neumann algebra with a faithful normal trace $\tau$, and $G$ a countable discrete group of automorphisms of $\mathscr{A}$ acting freely and preserving $\tau$ invariant. Then the lattice of all full subgroups $K$ of $[G]$ and the lattice of all intermediate von Neumann subalgebras $\mathscr{B}$ of $G \otimes \mathscr{A}$ are isomorphic by associating with each full subgroup $K$ the intermediate subalgebra

$$
\mathscr{B}(K)=\mathscr{R}\left[U \mid \phi_{U} \in K\right]
$$

and with each intermediate subalgebra $\mathscr{B}$ the full group

$$
K(\mathscr{B})=\left[\phi_{U} \mid U \in \mathscr{U}(G \otimes \mathscr{A}, \mathscr{A}) \cap \mathscr{B}\right] .
$$

Proof. The subalgebra $\mathscr{B}(K(\mathscr{B}))$ associated with the full group : $K(\mathscr{B})$ is the original $\mathscr{B}$. In fact, by Corollary 1 of Lemma 8 ,

$$
\begin{aligned}
\mathscr{B}(K(\mathscr{B})) & =\mathscr{R}\left[U \mid \phi_{U} \in K(\mathscr{B})\right] \\
& =\mathscr{R}[\mathscr{U}(G \otimes \mathscr{A}, \mathscr{A}) \cap \mathscr{B}]=\mathscr{B} .
\end{aligned}
$$

On the other hand, by the same discussion as the proof of [8; Proposition 6.1], it follows that the full group $K(\mathscr{B}(K))$ associated with the intermediate subalgebra $\mathscr{B}(K)$ is the original $K$. 
The one-to-one correspondence thus established between two lattices evidently preserves the order and consequently it is the lattice isomorphism.

4. Galois correspondence. In this section, we transfer the Dye correspondence stated in the preceding section to the commutant, then it gives the Galois correspondence under appropriate conditions.

Let $\mathscr{A}$ be a von Neumann algebra and $G$ a countable group of automorphisms acting freely on $\mathscr{A}$. We assume that $\mathscr{A}$ is represented on a Hilbert space $\mathscr{H}$ and each $g \in G$ is represented by a unitary operator $U_{g}$ on $\mathscr{H}: g(A)=U_{g} A U_{g}^{*}$ for $A \in \mathscr{A}$. The existence of such a representation of an arbitrary $\mathscr{A}$ is known by M. Henle [9; Lemma 1.4].

Lemma 9. (Cf. [12; Lemma 1]). If a unitary operator $U$ on $\mathscr{H}$ induces a freely acting automorphism of $\mathscr{A}$, then $U$ induces also a freely acting automorphism of $\mathscr{A}^{\prime}$.

Proof (by Henle). If $A^{\prime} \in \mathscr{A}^{\prime}$, then for $A \in \mathscr{A}$

$$
\left(U A^{\prime} U^{*}\right) A=U A^{\prime}\left(U^{*} A U\right) U^{*}=U\left(U^{*} A U\right) A^{\prime} U^{*}=A\left(U A^{\prime} U^{*}\right),
$$

showing that $U A^{\prime} U^{*} \in \mathscr{A}^{\prime}$. Thus $U$ induces an automorphism of $\mathscr{A}^{\prime}$. To show the free action, suppose that $A^{\prime} \in \mathscr{A}^{\prime}$ satisfies

$$
A^{\prime} B^{\prime}=\left(U B^{\prime} U^{*}\right) A^{\prime} \quad \text { for all } B^{\prime} \in \mathscr{A}^{\prime} \text {. }
$$

Then $\left(U^{*} A^{\prime}\right) B^{\prime}=B^{\prime}\left(U^{*} A^{\prime}\right)$ or $U^{*} A^{\prime} \in \mathscr{A}$. So, for $C \in \mathscr{A}$

$$
\left(U^{*} A^{\prime}\right) C=U^{*} C A^{\prime}=\left(U^{*} C U\right) U^{*} A^{\prime} \text {. }
$$

Since $U^{*}$ as well as $U$ induces a freely acting automorphism of $\mathscr{A}$, $U^{*} A^{\prime}=0$, hence $A^{\prime}=0$.

According to this lemma, we may consider $G$ as a group of automorphisms acting freely on the commutant $\mathscr{A}^{\prime}$. Thus, if $\alpha$ in $[G]$ has the form

$$
\alpha(A)=\sum_{g} P_{g} g\left(V A V^{*}\right)=\sum_{g} P_{g} U_{g} V A V^{*} U_{g}^{*} \quad \text { for } A \in \mathscr{A},
$$

where $V \in \mathscr{A}_{u}, \alpha$ induces an automorphism on $\mathscr{A}^{\prime}$ such that

$$
\alpha\left(A^{\prime}\right)=\sum_{g} P_{g} U_{g} V A^{\prime} V^{*} U_{g}^{*}=\sum_{g} P_{g} g\left(A^{\prime}\right) \quad \text { for } A^{\prime} \in \mathscr{A}^{\prime} .
$$

Let us denote by $[G]_{x}$ the set of all automorphisms of $\mathscr{A}^{\prime}$ having the form (6). In this representation, inner automorphisms contained in the representation of elements in $[G]$ disappear, hence $[G]_{\mathscr{X}}$ coincides exactly with the collection of all automorphisms of $\mathscr{A}^{\prime}$ given as a full group in the sense of Dye. Hence $[G]_{x}$ is a group. We say $[G]_{x}$ the centrally full group or, in short, the $\mathscr{\digamma}$-full group determined by $G$. It is somewhat 
interesting that the intact concept of full group introduced for abelian algebras has a utility for non-abelian $\mathscr{A}^{\prime}$, while we need the generalization for $\mathscr{A}$. This is the reason why $M$. Henle could get through his Galois theory without the generalized concept of full group.

In the following, we shall exchange $\mathscr{A}$ and $\mathscr{A}^{\prime}$ with each other in the above discussion. Let $\mathscr{A}$ be a von Neumann algebra on a Hilbert space $\mathscr{H}$. We assume that the commutant $\mathscr{A}^{\prime}$ is finite and has a faithful normal $G$-invariant trace. We construct the cross product $G \otimes \mathscr{A}^{\prime}$ on $G \otimes \mathscr{H}$. Then

LEMma 10. Let $\mathscr{B}^{\prime}$ be an intermediate subalgebra of $G \otimes \mathscr{A}^{\prime}$ and $\mathscr{B}$ be its commutant on $G \otimes \mathscr{H}$. Then $\mathscr{B}$ is the fixed subalgebra of $\left(1 \otimes \mathscr{A}^{\prime}\right)^{\prime}$ under the $\mathscr{\Sigma}$-full subgroup $K_{\mathscr{X}}(\mathscr{B})$; that is,

$$
\mathscr{B}=\left[A \in\left(1 \otimes \mathscr{A}^{\prime}\right)^{\prime} \mid \alpha(A)=A \text { for all } \alpha \in K\left(\mathscr{B}^{\prime}\right)\right] \text {. }
$$

Proof. Every $B$ in $\mathscr{B}$ trivially satisfies $U B U^{*}=B$ for each $U \in$ $\mathscr{U}\left(G \otimes \mathscr{A}^{\prime}, \mathscr{A}^{\prime}\right) \cap B^{\prime}$. Hence $\alpha(B)=B$ for each $\alpha \in K\left(\mathscr{B}^{\prime}\right)$. Conversely, let $C$ in $\left(1 \otimes \mathscr{A}^{\prime}\right)^{\prime}$ satisfy $\alpha(C)=C$ for each $\alpha \in K\left(\mathscr{B}^{\prime}\right)$, then $U C U^{*}=C$ for each $U \in \mathscr{U}\left(G \otimes \mathscr{A}, \mathscr{A}^{\prime}\right) \cap \mathscr{B}^{\prime}$. Since $\mathscr{U}\left(G \otimes \mathscr{A}^{\prime}, \mathscr{A}^{\prime}\right) \cap \mathscr{B}^{\prime}$ generates $\mathscr{B}^{\prime}$ (Corollary to Lemma 8), it follows $C \in \mathscr{B}$.

By Theorem 2, the Dye correspondence gives the lattice isomorphism between intermediate subalgebras of $G \otimes \mathscr{A}^{\prime}$ and full subgroups of $[G]$. Naturally, by taking commutants, we get the dual isomorphism between the lattice of all intermediate subalgebra $\mathscr{B}^{\prime}$ between $G \otimes \mathscr{A}^{\prime}$ and $1 \otimes \mathscr{A}^{\prime}$ and that of all von Neumann subalgebras $\mathscr{B}$ between $\left(G \otimes \mathscr{A}^{\prime}\right)^{\prime}$ and $\left(1 \otimes \mathscr{A}^{\prime}\right)^{\prime}$. On the other hand, by the correspondence

$$
K\left(\mathscr{B}^{\prime}\right) \longleftrightarrow K_{\mathscr{x}}(\mathscr{B})
$$

stated above, the lattice of full subgroups of $[G]$ for $1 \otimes \mathscr{A}^{\prime}$ is isomorphic to that of $\mathscr{z}$-full subgroups of $[G]_{\mathscr{x}}$ for $\left(1 \otimes \mathscr{A}^{\prime}\right)^{\prime}$. Hence we know that the lattice of all von Neumann subalgebras $\mathscr{B}$ between $\left(1 \otimes \mathscr{A}^{\prime}\right)^{\prime}$ and $\left(G \otimes \mathscr{A}^{\prime}\right)^{\prime}$ is dually isomorphic to that of $\mathscr{Z}$-full subgroups of $[G]_{x}$. Lemma 10 gives this correspondence concretely as $\mathscr{Z}$-full subgroup $K \leftrightarrow$ fixed algebra $\mathscr{B}$. Thus we get the following theorem.

THeOREm 3. Let $\mathscr{A}$ be a von Neumann algebra on $\mathscr{H}$ having the finite commutant $\mathscr{A}^{\prime}$ with a faithful normal trace $\tau$, and $G$ a countable group of automorphisms acting freely on $\mathscr{A}$. Suppose that each $g$ in $G$ is represented by a unitary operator $U_{g}$ on $\mathscr{H}$ and preserves $\tau$ invariant. Then, the lattice of all $\mathscr{z}$-full subgroups of $[G]_{x}$ for $\left(1 \otimes \mathscr{A}^{\prime}\right)^{\prime}$ and that of all intermediate von Neumann subalgebras between $\left(1 \otimes \mathscr{A}^{\prime}\right)^{\prime}$ and $\left(G \otimes \mathscr{A}^{\prime}\right)^{\prime}$ are dually isomorphic under correspondence which associate with 
each $\mathscr{2}$-full subgroup $K$ the intermediate subalgebra $\mathscr{B}$ invariant under $K$ in element-wise.

We regard the correspondence asserted in Theorem 3 as a prototype of Galois correspondence. Bearing this in mind, we give the definition of Galois extension as follows.

DEFINITION 4. Let $G$ be a countable group of automorphisms acting freely on $\mathscr{A}$ and $\mathscr{B}$ the fixed subalgebra of $\mathscr{A}$ under $G$. Then $\mathscr{A}$ is a Galois extension of $\mathscr{B}$ with Galois group $G$, if there exists a representation of $\mathscr{A}$ on some Hilbert space $\mathscr{K}$ satisfying the following conditions:

(i) $G$ has a unitary representation $U_{g}$ on $\mathscr{K}$ such that $g(A)=U_{g} A U_{g}^{*}$ for $A \in \mathscr{A}$

(ii) $\mathscr{A}^{\prime}$ is finite and has a faithful normal trace $\tau$ invariant under G.

(iii) $\mathscr{B}^{\prime}$ is algebraically isomorphic with the cross product $G \otimes \mathscr{A}^{\prime}$ by an isomorphic mapping such that $\mathscr{A}^{\prime} \leftrightarrow 1 \otimes \mathscr{A}^{\prime}$ and $U_{g} \leftrightarrow g \otimes I$.

Then we get the Fundamental Theorem of Galois Theory in the following form.

THEOREM 4. If $\mathscr{A}$ is a Galois extension of $\mathscr{B}$ with Galois group $G$, then the lattice of all intermediate von Neumann subalgebras between $\mathscr{A}$ and $\mathscr{B}$ and that of all $\mathscr{Z}$-full subgroups of $[G]_{x}$ are dually isomorphic under the Galois correspondence which associate with each $\mathscr{\Sigma}$-full subgroup $K_{\mathscr{x}}$ the intermediate subalgebra $\mathscr{C}$ invariant under $K_{\mathscr{x}}$ in element-wise.

Proof. Let $\mathscr{K}$ be the Hilbert space on which $\mathscr{A}$ is represented as shown in the definition of Galois extension. Then by Theorem 2 every intermediate subalgebra $\mathscr{D}$ of $G \otimes \mathscr{A}^{\prime}$ is generated by

$$
\mathscr{L}\left(G \otimes \mathscr{A}^{\prime}, \mathscr{A}^{\prime}\right) \cap \mathscr{D} .
$$

By the definition of Galois extension, $\mathscr{B}^{\prime}$ is algebraically isomorphic to $G \otimes \mathscr{A}^{\prime}$. We denote the isomorphism by $\theta: \theta\left(G \otimes \mathscr{A}^{\prime}\right)=\mathscr{B}^{\prime}$. Clearly every intermediate von Neumann subalgebra $\mathscr{C}^{\prime}$ between $\mathscr{B}^{\prime}$ and $\mathscr{A}^{\prime}$ is the isomorphic image $\theta(\mathscr{D})$ of an intermediate subalgebra $\mathscr{D}$ of $G \otimes \mathscr{A}^{\prime}$. The set of automorphisms $\alpha$ of $\mathscr{A}^{\prime}$ such that $\alpha\left(A^{\prime}\right)=\theta(U) A^{\prime} \theta\left(U^{*}\right)$, $\left(U \in \mathscr{C}\left(G \otimes \mathscr{A}^{\prime}, \mathscr{A}^{\prime}\right)\right)$ is the full group $[G]$ for $\mathscr{A}^{\prime}$ on $\mathscr{K}$ and the set of automorphisms $\alpha$ of $\mathscr{A}$ such that $\alpha(A)=\theta(U) A \theta\left(U^{*}\right)$ is the $\mathscr{Z}$-full group $[G]_{\mathscr{X}}$ for $\mathscr{A}$ on $\mathscr{K}$. Put 


$$
K=\left\{\alpha \mid \alpha\left(A^{\prime}\right)=\theta(U) A^{\prime} \theta\left(U^{*}\right), U \in \mathscr{L}\left(G \otimes \mathscr{A}^{\prime}, \mathscr{A}^{\prime}\right) \cap \mathscr{D}\right\}
$$

and

$$
K_{\mathscr{R}}=\left\{\alpha \mid \alpha(A)=\theta(U) A \theta\left(U^{*}\right), U \in \mathscr{U}\left(G \otimes \mathscr{A}^{\prime}, \mathscr{A}^{\prime}\right) \cap \mathscr{D}\right\} .
$$

Then $K$ is the full subgroup of $[G]$ corresponding to $\mathscr{C}^{\prime}=\theta(\mathscr{D})$ and the commutant $\mathscr{C}$ of $\mathscr{C}^{\prime}$ is the algebra of all elements invariant under $K_{\mathscr{x}}$ since $\mathscr{C}^{\prime}=\mathscr{R}\left[\mathscr{C}\left(\mathscr{B}^{\prime}, \mathscr{A}^{\prime}\right) \cap \mathscr{C}^{\prime}\right]$. Thus we get the correspondence between $\mathscr{Z}$-full subgroups $K_{\mathscr{X}}$ of $[G]_{\mathscr{X}}$ and fixed subalgebras $\mathscr{C}$ of $\mathscr{A}$. The dual isomorphism of this correspondence is clear.

Now, we give an example of Galois extension. Let $\Gamma$ be a finite von Neumann algebra acting standardly on $\mathscr{C}([6 ;$ I. 5.5]), and $G$ be a countably infinite group of automorphisms acting freely on $\Gamma$ and preserving a faithful normal trace invariant. Construct the cross product $G \otimes \Gamma^{\prime}$ on the Hilbert space $\mathscr{K}=G \otimes \mathscr{C}$, then $1 \otimes \Gamma^{\prime}$ and hence $G \otimes \Gamma^{\prime}$ are finite. Each $g$ in $G$ is represented by the unitary operator $g \otimes I$. We take $\mathscr{A}=$ $\left(1 \otimes \Gamma^{\prime}\right)^{\prime}$ and $\mathscr{A}^{\prime}=1 \otimes \Gamma^{\prime}$. Then $G$ can be considered as a group of automorphisms of $\mathscr{A}$ (resp. $\left.\mathscr{A}^{\prime}\right)$ by the automorphisms induced by $g \otimes I$ $(g \in G)$ on $\mathscr{A}\left(\right.$ resp. $\left.\mathscr{A}^{\prime}\right)$. Let $\mathscr{B}=\mathscr{A}^{a}$ be the fixed subalgebra of $\mathscr{A}$ under this group $G$. Clearly $\mathscr{A}^{\prime}$ is isomorphic to $\Gamma^{\prime}$, hence it is finite and has a trace invariant under $G$. We may construct $G \otimes \mathscr{A}^{\prime}=$ $G \otimes\left(1 \otimes \Gamma^{\prime}\right)$ on $G \otimes \mathscr{K}$. Since the cross product is independent of the Hilbert space used to construct it, this cross product is algebraically isomorphic with $\mathscr{B}^{\prime}=G \otimes \Gamma^{\prime}$. Thus $\mathscr{A}$ satisfies all of the three conditions of Galois extension. $\mathscr{A}$ is the Galois extension of $\mathscr{B}$ with Galois group G. In addition, $\mathscr{A}=\left(1 \otimes \Gamma^{\prime}\right)^{\prime} \cong I_{\infty} \otimes \Gamma$ is a properly infinite von Neumann algebra. Thus we get an example of properly infinite Galois extension.

Of course, if $G$ is finite in above, $\mathscr{A}$ gives an example of finite algebra with a finite Galois group. For finite Galois group, we get the following in general.

Lemma 11. If $G$ is a finite group acting freely on a semifinite von Neumann algebra $\mathscr{A}$, then $\mathscr{A}$ is the Galois extension of $\mathscr{B}=\mathscr{A}^{G}$ with the finite Galois group G.

PRoof. (Essentially due to [9] and [13]). There exists such a representation of $\mathscr{A}$ that $\mathscr{A}^{\prime}$ is finite. Then $\mathscr{A}^{\prime}$ has an invariant trace, since $G$ is finite. Using the Hilbert space $\mathscr{H}$ of this representation, construct the cross product $G \otimes \mathscr{A}$ on $G \otimes \mathscr{H}$. Then $(1 \otimes \mathscr{A})^{\prime} \cong I_{m} \otimes \mathscr{A}^{\prime}$ is finite where $m=\operatorname{order}(G)$ and each automorphism $g$ in $G$ is represented by the unitary operator $g \otimes I$ on $G \otimes \mathscr{H}$. Therefore, we may assume 
that $\mathscr{A}$ is represented on a Hilbert space $\mathscr{K}$ in such a way that $\mathscr{A}^{\prime}$ is finite and the group $G$ is freely acting on both $\mathscr{A}$ and $\mathscr{A}^{\prime}$ (Lemma 9). Construct the cross product $G \otimes \mathscr{A}^{\prime}$ on $G \otimes \mathscr{K}$. We may decompose $\mathscr{K}$ into a direct sum $\left[\mathscr{A}^{\prime} \xi_{1}\right] \oplus\left[\mathscr{L}^{\prime} \xi_{2}\right] \oplus \cdots$ of orthogonal subspaces on each of which $\mathscr{A}^{\prime}$ has a generating vector $\xi_{i}$ where $\xi_{i}$ are invariant under $U_{g}$ $(g \in G)$. In fact, for non-zero vector $\eta_{1} \in \mathscr{H}, \xi_{1}=\sum_{h \in G} U_{h} \xi_{1}$ is invariant under $U_{g}$. Take an $\eta_{2} \in\left[\mathscr{A}^{\prime} \xi_{1}\right]^{\perp}$, then

$$
\begin{aligned}
\left\langle A^{\prime} \xi_{1}, U_{g} \eta_{2}\right\rangle & =\left\langle U_{g}^{*} A^{\prime} \xi_{1}, \eta_{2}\right\rangle \\
& =\left\langle g^{-1}\left(A^{\prime}\right) U_{g}^{*} \xi_{1}, \eta_{2}\right\rangle=\left\langle g^{-1}\left(A^{\prime}\right) \xi_{1}, \eta_{2}\right\rangle .
\end{aligned}
$$

Hence $\xi_{2}=\sum_{h} U_{h} \eta_{2} \in\left[\mathscr{A}^{\prime} \xi_{1}\right]^{\perp}$ is invariant under $U_{g}$ and $\left[\mathscr{A}^{\prime} \xi_{1}\right]$ and $\left[\mathscr{A}^{\prime} \xi_{2}\right]$ are mutually orthogonal subspaces of $\mathscr{K}$. Continuing this process, we get the desired decomposition of $\mathscr{K}$. The vectors $\xi_{i}$ may be considered as normalizing vectors. Now, take

$$
\zeta_{i}=\frac{1}{\sqrt{m}} \sum_{a \in G} a \otimes \xi_{i} \in G \otimes\left[\mathscr{A}^{\prime} \xi_{i}\right],
$$

and define an operator $V_{i}: \mathscr{A}^{\prime} \xi_{i} \rightarrow\left(1 \otimes \mathscr{A}^{\prime}\right) \zeta_{i}$ by

$$
V_{i}\left(A^{\prime} \xi_{i}\right)=\left(1 \otimes A^{\prime}\right) \zeta_{i} \text {. }
$$

Then

$$
\begin{aligned}
\left\|\left(1 \otimes A^{\prime}\right) \zeta_{i}\right\|_{G \otimes \mathscr{C}}^{2} & =\left\|\frac{1}{\sqrt{m}} \sum_{a \in G}\left(1 \otimes A^{\prime}\right)\left(a \otimes \xi_{i}\right)\right\|_{G \otimes \mathscr{C}}^{2} \\
& =\frac{1}{m}\left\|\sum_{a} a \otimes a\left(A^{\prime}\right) \xi_{i}\right\|_{G \otimes \mathscr{C}}^{2} \\
& =\frac{1}{m} \sum_{a}\left\|a\left(A^{\prime}\right) \xi_{i}\right\|_{\mathscr{C}}^{2}=\frac{1}{m} \sum_{a}\left\|A^{\prime} \xi_{i}\right\|_{\mathscr{\mathscr { C }}}^{2} \\
& =\left\|A^{\prime} \xi_{i}\right\|_{\mathscr{K}}^{2},
\end{aligned}
$$

so $V_{i}$ may be extended to an isometry of $\left[\mathscr{A}^{\prime} \xi_{i}\right]$ onto $\mathscr{C}_{i}=\left[\left(1 \otimes \mathscr{A}^{\prime}\right) \zeta_{i}\right]$ in $G \otimes \mathscr{K}$. Put $U=\sum_{i} V_{i}$. Then $U$ is an isometry of $\mathscr{K}$ to $\mathscr{K}=$ $\mathscr{H}_{1} \oplus \mathscr{H}_{2} \oplus \cdots$. For $A^{\prime} \in \mathscr{A}^{\prime}, g \in G$,

$$
\begin{aligned}
(g \otimes I)\left(1 \otimes A^{\prime}\right) \zeta_{i} & =(g \otimes I)\left(\frac{1}{\sqrt{m}} \sum_{a} a \otimes a\left(A^{\prime}\right) \xi_{i}\right) \\
& =\frac{1}{\sqrt{m}} \sum_{a} a g^{-1} \otimes a\left(A^{\prime}\right) \xi_{i}=\frac{1}{\sqrt{m}} \sum_{b} b \otimes b g\left(A^{\prime}\right) \xi_{i} \\
& =\left(1 \otimes g\left(A^{\prime}\right)\right)\left(\frac{1}{\sqrt{m}} \sum_{b} b \otimes \xi_{i}\right) \\
& =\left(1 \otimes g\left(A^{\prime}\right)\right) \zeta_{i} \in\left(1 \otimes \mathscr{A}^{\prime}\right) \zeta_{i} .
\end{aligned}
$$


In other words $g \otimes I$ maps $\left(1 \otimes \mathscr{A}^{\prime}\right) \zeta_{i}$ into itself for all $i$. Hence it maps $\mathscr{K}$ into itself, so the projection $P: G \otimes \mathscr{K} \rightarrow \mathscr{C}$ commutes with $g \otimes I$. Therefore $P \in\left(G \otimes \mathscr{A}^{\prime}\right)^{\prime}$. By the definition of $U$, the mapping $T \rightarrow U T U^{*}$ of $\mathscr{L}(\mathscr{K})$ onto $\mathscr{L}(\mathscr{M})$ takes $\mathscr{A}^{\prime}$ onto $P\left(1 \otimes \mathscr{A}^{\prime}\right)$. In addition

$$
\begin{aligned}
U U_{g} U^{*}\left(1 \otimes A^{\prime}\right) \zeta_{i} & =U U_{g}\left(A^{\prime} \xi_{i}\right)=U g\left(A^{\prime}\right) \xi_{i} \\
& =\left(1 \otimes g\left(A^{\prime}\right)\right) \zeta_{i}=(g \otimes I)\left(1 \otimes A^{\prime}\right) \zeta_{i}
\end{aligned}
$$

for all $i$, so that $U$ induces a spatial isomorphism of $\mathscr{B}^{\prime}$ onto

$$
\left.\left(G \otimes \mathscr{A}^{\prime}\right)\right|_{\mathscr{A}}=P\left(G \otimes \mathscr{A}^{\prime}\right) P .
$$

Therefore, if we prove $C(P)=I$, then $\mathscr{B}^{\prime} \cong G \otimes \mathscr{A}^{\prime}$ by [6; I. 2. Proposition 2].

To show $C(P)=I$, notice that the center of $1 \otimes \mathscr{A}^{\prime}$ is $1 \otimes \mathscr{Z}$. Hence, by [6; I. 1. Corollaire 2 de Proposition 7], it suffices to show that

$$
\sum_{i} \oplus\left[(1 \otimes \mathscr{Z})^{\prime} \zeta_{i}\right]=G \otimes \mathscr{K} \text {. }
$$

Let $P_{g}$ be the projection from $G \otimes \mathscr{K}$ to $g \otimes \mathscr{K}$. Then, for any $Z \in \mathscr{Z}$,

$$
P_{g}(1 \otimes Z)\left(\sum_{a} a \otimes \xi_{a}\right)=P_{g}\left(\sum_{a} a \otimes a(Z) \xi_{a}\right)=g \otimes g(Z) \xi_{g}
$$

and

$$
(1 \otimes Z) P_{g}\left(\sum_{a} a \otimes \xi_{a}\right)=(1 \otimes Z)\left(g \otimes \xi_{g}\right)=g \otimes g(Z) \xi_{g} .
$$

It follows $P_{g} \in(1 \otimes \mathscr{Z})^{\prime}$. Hence,

$$
g \otimes \xi_{i}=\sqrt{m} P_{g} \zeta_{i} \in(1 \otimes \mathscr{Z})^{\prime} \zeta_{i}
$$

for any $g \in G$ and $i=1,2, \cdots$, and hence

$$
g \otimes A^{\prime} \xi_{i}=\left(1 \otimes g^{-1}\left(A^{\prime}\right)\right)\left(g \otimes \xi_{i}\right) \in(1 \otimes \mathscr{Z})^{\prime} \zeta_{i} .
$$

As $\left[\mathscr{A}^{\prime} \xi_{i}\right]_{i=1,2, \ldots}$ generate $\mathscr{K}$, it follows that

$$
\sum_{i} \oplus\left[(1 \otimes \mathscr{Z})^{\prime} \zeta_{i}\right]=G \otimes \mathscr{K},
$$

completing the proof.

For finite von Neumann algebras, we get conversely

Lemma 12. Let $\mathscr{A}$ be a finite von Neumann algebra and $G$ be freely acting on $\mathscr{A}$. Then, if $\mathscr{A}$ is a Galois extension of $\mathscr{B}=\mathscr{A}^{G}, G$ is a finite group.

Proof. We owe this proof essentially to Henle. By assumption, $\mathscr{A}$ is represented on some Hilbert space $\mathscr{K}$ in such a way that $\mathscr{A}^{\prime}$ is finite and $\mathscr{B}^{\prime} \cong G \otimes \mathscr{A}^{\prime}$. Let $\theta$ be the isomorphic mapping of $G \otimes \mathscr{A}^{\prime}$ onto 
$\mathscr{B}$. Fix a non-zero vector $\eta$ in $\mathscr{K}$ and define a normal positive linear functional $\omega$ on $G \otimes \mathscr{A}^{\prime}$ by

$$
\omega(T)=\langle\theta(T) \eta, \eta\rangle_{\mathscr{x}} \quad\left(T \in G \otimes \mathscr{A}^{\prime}\right) .
$$

Suppose that $\mathscr{A}^{\prime}$ is represented on another Hilbert space $\mathscr{H}$ with separating and generating vector $\xi$. Then $G \otimes \mathscr{A}^{\prime}$ on $G \otimes \mathscr{H}$ has a separating and generating vector $1 \otimes \xi$. By [6; III. 1. Théorème 4], there exists a vector $\zeta \in G \otimes \mathscr{H}$ such that

$$
\omega(T)=\langle T \zeta, \zeta\rangle_{G \otimes \mathscr{C}}
$$

for $T \in G \otimes \mathscr{A}^{\prime}$.

Define an operator $U:\left(G \otimes \mathscr{A}^{\prime}\right) \zeta \rightarrow \mathscr{B}^{\prime} \eta$ by

$$
U(T \zeta)=\theta(T) \eta \quad \text { for } T \in G \otimes \mathscr{A}^{\prime} .
$$

The computation

$$
\begin{aligned}
\|\theta(T) \eta\|_{\mathscr{X}}^{2} & =\left\langle\theta\left(T^{*} T\right) \eta, \eta\right\rangle_{\mathscr{x}}=\omega\left(T^{*} T\right) \\
& =\left\langle T^{*} T \zeta, \zeta\right\rangle_{G \otimes \mathscr{X}}=\|T \zeta\|_{* \otimes \mathscr{C}}^{2}
\end{aligned}
$$

shows that $U$ is an isometry, hence $U$ may be extended to a unitary operator from $\mathscr{K}=\left[\left(G \otimes \mathscr{A}^{\prime}\right) \zeta\right]$ in $G \otimes \mathscr{C}$ onto $\mathscr{N}=\left[\mathscr{B}^{\prime} \eta\right]$ in $\mathscr{K}$. Let $P$ be the projection of $G \otimes \mathscr{H}$ onto $\mathscr{M}$ and $Q$ be the one of $\mathscr{K}$ onto $\mathscr{N}$. As $\mathscr{C}$ is invariant under $G \otimes \mathscr{A}^{\prime}, P \in\left(G \otimes \mathscr{A}^{\prime}\right)^{\prime}$. Similarly $Q \in \mathscr{B}$. Then the operator $U$ induces a spatial isomorphism of $\left.\left(G \otimes \mathscr{A}^{\prime}\right)\right|_{\mathscr{M}}$ onto $\left.\mathscr{B}^{\prime}\right|_{\mathscr{r}}$. Furthermore, for $T \in G \otimes \mathscr{A}^{\prime}, S \in \mathscr{B}^{\prime}$,

$$
\left(U T U^{*}\right)(S \eta)=U T\left(\theta^{-1}(S) \zeta\right)=U\left(T \theta^{-1}(S) \zeta\right)=\theta(T)(S \eta)
$$

and $\theta$ maps $1 \otimes \mathscr{A}^{\prime}$ onto $\mathscr{A}^{\prime}$, therefore $U$ induces a spatial isomorphism of $\left.\left(1 \otimes \mathscr{A}^{\prime}\right)\right|_{\mathscr{A}}$ onto $\left.\mathscr{A}^{\prime}\right|_{\mathscr{r}}$. Then $U$ also induces a spatial isomorphism

$$
\left(1 \otimes \mathscr{A}^{\prime}\right)^{\prime} P=\left(\left.\left(1 \otimes \mathscr{A}^{\prime}\right)\right|_{\mathscr{R}}\right)^{\prime} \longleftrightarrow\left(\left.\mathscr{A}^{\prime}\right|_{\mathscr{r}}\right)^{\prime}=\left.\mathscr{A}\right|_{\mathscr{r}}
$$

Let $P_{g}^{\prime}$ be the projection of $G \otimes \mathscr{H}$ onto $g \otimes \mathscr{H} . \quad$ As $g \otimes \mathscr{H}$ is invariant under $1 \otimes \mathscr{A}^{\prime}, P_{g}^{\prime} \in\left(1 \otimes \mathscr{A}^{\prime}\right)^{\prime}$. Therefore $P_{g}=\left.U P P_{g}^{\prime} P U^{*} \in \mathscr{A}\right|_{\mathscr{r}}$.

$$
\begin{aligned}
g\left(P_{1}\right) & =U_{g} P_{1} U_{g}^{*}=\left(U(g \otimes 1) U^{*}\right) U P P_{1}^{\prime} P U^{*}\left(U(g \otimes 1)^{*} U^{*}\right) \\
& =U\left((g \otimes 1) P P_{1}^{\prime} P(g \otimes 1)^{*}\right) U^{*}=U P(g \otimes 1) P_{1}^{\prime}(g \otimes 1)^{*} P U^{*} \\
& =U P P_{g^{-1}}^{\prime} P U^{*}=P_{g-1}
\end{aligned}
$$

and

$$
\sum_{g \in G} P_{g}=\sum_{g} U P P_{g}^{\prime} P U^{*}=U P\left(\sum_{g} P_{g}^{\prime}\right) P U^{*}=U P U^{*} .
$$

Let $\delta=\tau\left(P_{1}\right)>0$, where $\tau$ is the canonical trace on $\left.\mathscr{A}\right|_{\mathscr{r}}$. Then for $g \in G$

$$
\tau\left(P_{g}\right)=\tau\left(g^{-1}\left(P_{1}\right)\right)=\delta,
$$


so

$$
\sum_{g \in G} \delta=\sum_{g} \tau\left(P_{g}\right)=\tau\left(\sum_{g} P_{g}\right)=\tau\left(U P U^{*}\right)<\infty,
$$

proving that $G$ is finite.

By these two lemmas, it becomes possible to give a clear-cut characterization of the Galois group for finite von Neumann algebras.

Theorem 5. (Cf. [13; Theorem 3]). If $\mathscr{A}$ is a finite von Neumann algebra, then a group $G$ acting freely on $\mathscr{A}$ is Galois if and only if it is finite.

Hence if $\mathscr{A}$ has a countably infinite Galois group, $\mathscr{A}$ is necessarily properly infinite. We gave already such an example of Galois extension.

5. Relation to Henle's Galois theory. In [9] M. Henle employed a different definition of Galois extension from ours. Given a von Neumann algebra $\mathscr{A}$ and a countable group of automorphisms acting freely on $\mathscr{A}$, he defines $\mathscr{A}$ to be a Galois extension of $\mathscr{B}=\mathscr{A}^{G}$ with Galois group $G$, if there exist mutually orthogonal projections $P_{g} \in \mathscr{A}(g \in G)$ such that $\sum_{g} P_{g}=I$ and $g\left(P_{h}\right)=P_{h g^{-1}}(g, h \in G)$. His purpose is to settle a condition to ensure $\mathscr{B}^{\prime} \cong G \otimes \mathscr{A}^{\prime}$. Indeed, his definition of Galois extension gives a sufficient condition for this isomorphism just as it is, ([9; Theorem 2.1]). On the other hand, we take this isomorphism in the definition of Galois extension as one of the conditions. Besides we assume the finiteness of $\mathscr{A}^{\prime}$. Hence purely infinite algebras are excluded completely in our Galois theory, but Henle does not place such a restriction on $\mathscr{A}$. However, owing to the finiteness of $\mathscr{A}^{\prime}$ we can use freely the natural expectation on arbitrary subalgebras of $G \otimes \mathscr{A}^{\prime}$, and so we get the Galois correspondence between all intermediate subalgebras of $\mathscr{A}$ and all $\mathscr{Z}$-full subgroups of $[G]_{x}$. On the contrary, Henle's theory insists only the Galois correspondence between subgroups of $G$ and their corresponding intermediate subalgebras of $\mathscr{A}$. Hence in our case, Henle's type of correspondence remains true without the finiteness of $\mathscr{A}^{\prime}$. To show this we need.

LEmma 13. Let $G$ be freely acting on $\mathscr{A}$. Then the lattice of all subgroup $K$ of $G$ is imbedded in the lattice of all full subgroups of [G] by the mapping $K \rightarrow[K]$.

Proof. If $K_{1}$ and $K_{2}$ are different subgroups of $G$, then $\left[K_{1}\right] \neq\left[K_{2}\right]$. In fact, we may assume that there exists an element $g_{1}$ in $K_{1}$ and not in $K_{2}$. It suffices to show that this $g_{1}$ is not in $\left[K_{2}\right]$. Suppose contrary, then since 


$$
\sup _{g_{2} \in K_{2}} F\left(g_{1}, g_{2}\right)=I,
$$

there exists such a $g_{2}$ in $K_{2}$ that $F\left(g_{1}, g_{2}\right) \neq 0$. This contradicts the free action of $G$. Therefore, all subgroups of $G$ are mapped into the lattice of all full subgroups of $[G]$ in one-to-one way by the above mapping.

We show that this mapping conserves the lattice operations.

$$
\left[K_{1} \vee K_{2}\right] \supset\left[K_{1}\right] \vee\left[K_{2}\right]
$$

is clear. Conversely, by $\left[K_{1}\right] \supset K_{1}$ and $\left[K_{2}\right] \supset K_{2}$, [ $\left.K_{1}\right] \vee\left[K_{2}\right] \supset K_{1} \vee K_{2}$ and hence $\left[K_{1}\right] \vee\left[K_{2}\right] \supset\left[K_{1} \vee K_{2}\right]$. Next, let $\alpha \in\left[K_{1}\right] \wedge\left[K_{2}\right]$, then $\alpha$ has representations

$$
\begin{aligned}
\alpha(A) & =\sum_{h \in K_{1}} P_{h} h\left(U A U^{*}\right) \\
& =\sum_{k \in K_{2}} P_{k} k\left(V A V^{*}\right)
\end{aligned}
$$

for all $A \in \mathscr{A}$. If $P_{h} P_{k} \neq 0, P_{h} P_{k} h\left(U A U^{*}\right)=P_{h} P_{k} k\left(V A V^{*}\right)$. Then, $h=$ $k \in K_{1} \wedge K_{2}$ on $P_{h} P_{k}$. In fact, if not so, substituting $U^{*} A U$ for $A$, it follows

$$
P_{h} P_{k} k^{-1} h(A)=P_{h} P_{k} V U^{*} A U V^{*}
$$

contrary to the free action of $k^{-1} h \neq 1$. Therefore $\alpha$ has a representation

$$
\alpha(A)=\sum_{g \in K_{1} \wedge K_{2}} P_{g} g\left(W A W^{*}\right)
$$

for all $A \in \mathscr{A}$. Hence $\alpha \in\left[K_{1} \wedge K_{2}\right]$. Therefore $\left[K_{1} \wedge K_{2}\right] \supset\left[K_{1}\right] \wedge\left[K_{2}\right]$. The converse inclusion is clear. Thus the proof is completed.

Now we assume a von Neumann algebra $\mathscr{A}$ and a group $G$ of freely acting automorphisms of $\mathscr{A}$ satisfy the conditions of Galois extension but the finiteness of $\mathscr{A}^{\prime}$. Hence $\mathscr{B}^{\prime} \cong G \otimes \mathscr{A}^{\prime}$ by the condition (iii) of Galois extension. But, in this case, the one-to-one correspondence between intermediate subalgebras of $G \otimes \mathscr{A}^{\prime}$ and full subgroups of $[G]$ is not certain since we cannot apply Theorem 3. However, let $K$ be a subgroup of $G$ and $K \otimes \mathscr{A}^{\prime}$ be the cross product of $K$ and $\mathscr{A}^{\prime}$, which is a subalgebra of $G \otimes \mathscr{A}^{\prime}$. Let $[K]$ be the full subgroup of $[G]$ determined by the subgroup $K$ corresponding to $K \otimes \mathscr{A}^{\prime}$. Then by Lemma 13, the correspondence $[K] \leftrightarrow K \otimes \mathscr{A}^{\prime}$ gives the one-to-one correspondence between subgroups $K$ of $G$ and subfamily of intermediate subalgebras $K \otimes \mathscr{A}^{\prime}$ of $G \otimes \mathscr{A}^{\prime}$ without the finiteness of $\mathscr{A}^{\prime}$. In fact, by the notation in $\S 3$, $[K]$ is the set of automorphism of $1 \otimes \mathscr{A}^{\prime}$ given by

$$
U \in \mathscr{N}\left(G \otimes \mathscr{A}^{\prime}\right) \cap\left(K \otimes \mathscr{A}^{\prime}\right) .
$$

On the other hand, since $K \otimes I$ and $1 \otimes \mathscr{A}_{u}^{\prime}$ are contained in $[K]$ and 
generates $K \otimes \mathscr{A}^{\prime}, \mathscr{R}\left[U \mid \phi_{U} \in[K]\right]=K \otimes \mathscr{A}^{\prime}$. Then this correspondence inherites to the correspondence between $\mathscr{Z}$-full subgroups $[K]_{\mathscr{x}}$ of $[G]_{x}$ and their fixed algebras of $\mathscr{A}$ by the same manner investigated in the preceding section. Furthermore, since the fixed algebra for the $\mathscr{Z}$ full subgroup $[K]_{x}$ coincides with that of the subgroup $K$ of $G$ acting on $\mathscr{A}$, we get the one-to-one correspondence between the family of subgroups of $G$ and their fixed subalgebras of $\mathscr{A}$. This is nothing but the correspondence given by M. Henle.

The remaining question is whether there exist full subgroups in $[G]$ not determined by a subgroup of $G$. This is answered by a theorem of measure preserving automorphisms. H. A. Dye has proved that any singly-generated infinite group $G$ of measure preserving automorphisms of a measure algebra is approximately finite, ([7; Theorem 1]). Since every non-trivial subgroup of $G$ is infinite, it is of type II in the sense of Dye. But, by the approximate finiteness of $G,[G]$ contains subgroups $\tilde{K}$ of type $I$. This implies that the full subgroup $[\widetilde{K}]$ determined by $\widetilde{K}$ is not determined by a subgroup of $G$.

REMARK. After the preparation of the present paper, we knew that D. Bures discussed the same notion with our local innerness in his recent work: Abelian subalgebras of von Neumann algebras, Memoirs of the Amer. Math. Soc., No 110 (1971).

\section{BIBLIOGRAPHY}

[1] W. Arveson, Analyticity in operator algebras, Amer. J. Math., 89 (1967) 578-642.

[2] H. BEHNCKE, Automorphisms of crossed products, Tôhoku Math. J., 21 (1969) 580-600.

[3] H. CHODA, On automorphisms of abelian von Neumann algebras, Proc. Japan Acad., 41 (1965) 280-283.

[4] M. CHODA, On the conditional expectation of a partial isometry in a certain von Neumann algebra, Proc. Japan Acad., 41 (1965) 277-279.

[5] J. Dixmier, Sous-anneaux abélians maximaux dans les facteurs de type fini, Ann. Math., 59 (1954) 279-286.

[6] J. Dixmier, Les Algèbres d'Opérateurs dans l'Espace Hilbertien, Gauthier-Villars, Paris (1957).

[7] H. A. DYE, On groups of measure preserving transformations I, Amer. J. Math., 81 (1959) $119-159$.

[8] H. A. DyE, On groups of measure preserving transformations II, Amer. J. Math., 85 (1963) 551-576.

[9] M. HenLe, Galois theory of $W^{*}$-algebras, to appear.

[10] R. R. Kallman, A generalization of free action, Duke Math. J., 36 (1969) 781-789.

[11] M. Nakamura and Z. TAKeda, On some elementary properties of the crossed products of von Neumann algebras, Proc. Japan Acad., 34 (1958) 489-494.

[12] M. Nakamura and Z. Takeda, A Galois theory for finite factors, Proc. Japan Acad., 36 (1960) 258-260. 
[13] M. Nakamura and Z. Takeda, On the fundamental theorem of the Galois theory for finite factors, Proc. Japan Acad., 36 (1960) 313-318.

[14] M. Nakamura and Z. Takeda, On inner automorphisms of certain finite factors, Proc. Japan Acad., 37 (1961) 31-32.

[15] J. von Neumann, On rings of operators III, Ann. Math., 41 (1940) 94-161.

[16] Z. TAKEDA, On the extension theorem of the Galois theory for finite factors, Proc. Japan Acad., 37 (1961) 78-82.

[17] Z. TAKEDA, On the normal basis theorem of the Galois theory for finite factors, Proc. Japan Acad., 37 (1961) 144-148.

[18] T. Turumaru, On the crossed product of operator algebras, Tôhoku Math. J., 10 (1958) $355-364$.

[19] H. UmegakI, Conditional expectation in an operator algebra, Tôhoku Math. J., 6 (1954) $177-181$.

[20] H. UMEGakI, Positive definite functions and direct product of Hilbert spaces, Tôhoku Math. J., 7 (1955) 206-211.

[21] G. Zeller-Meier, Produits croises d'une $C^{*}$-algèbre par un groupe d'automorphisms, J. de Math. Pures et Appl., 47 (1968) 101-239.

FACULTY OF ENGINEeRING

IBARAKI UNIVERSITY

HITACHI, JAPAN. 